\title{
The Gateway Theory: How Regional Neural Activation Creates a Gateway for Immune Cells via an Inflammation Amplifier
}

\author{
Hideki Ogura, Yasunobu Arima, Daisuke Kamimura, Masaaki Murakami
}

\begin{abstract}
The inflammation amplifier, a nuclear factor-kappa B (NF-kB) feedback loop in non-immune cells including fibroblasts and endothelial cells, describes how NF-kB-mediated transcriptions are enhanced to induce the inflammation in the presence of signal Tranducer and Activator of Transcription 3 (STAT3) activation. It was originally discovered in rheumatoid arthritis and multiple sclerosis mouse models and has since been shown to be associated with various human diseases and disorders including autoimmune diseases, metabolic syndromes, neurodegenerative diseases, and other inflammatory diseases. The amplifier begins with IL-17, which acts as the main signal to express NF-kB-mediated transcriptions, and IL-6, an NF-kB target, which functions as a fuel for the inflammation amplifier. Indeed, other NF-kB targets including various chemokines also act as effector molecules that cause local accumulation of various immune cells and subsequent inflammation. Through extensive

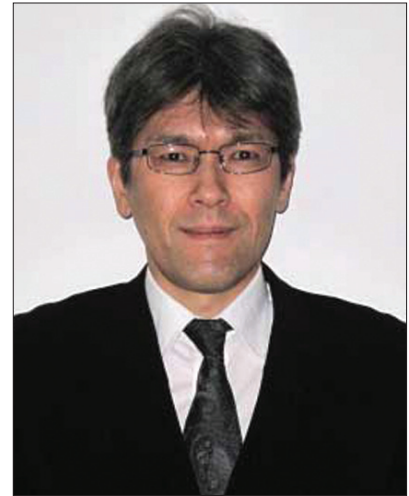

Dr. Masaaki Murakami studies in the multiple sclerosis model experimental autoimmune encephalomyelitis, we recently demonstrated that regional neural activation induces excess activation of the inflammation amplifier at specific blood vessels in the fifth lumbar cord, creating a gateway for immune cells to enter the central nervous system (CNS). We thus propose the gateway theory to describe how regional neural activation enables immune cells to enter the CNS from the blood and argue that this theory might provide novel therapeutic targets for inflammatory diseases and disorders. (Biomed $J$ 2013;36:269-273)
\end{abstract}

Key words: blood-brain barrier, chemokines, gateway theory, inflammation amplifier, Th17

\section{Discovery of the inflammation amplifier in non-immune cells}

$\mathrm{T}$ helper 17 (Th17) cells are IL-17 producers that have roles in many autoimmune diseases including rheumatoid arthritis and multiple sclerosis. ${ }^{[1-4]}$ IL-17 has been shown to contribute to the pathologies of the F759 mouse, a rheumatoid arthritis model that expresses a mutant variant of the IL-6 signaling transducer gp130 (Y759F) shows enhanced IL-6-mediated STAT3 activation due to a lack of SOCS3-mediated suppression. ${ }^{[3]}$ Because in F759 mice serum IL-17 concentrations and Th17 cells increase with age, and IL-17 deficiency suppresses disease development, we concluded that the arthritis is IL-17 dependent. Forced expression of IL-17 in F759 mice increased only one cyto- kine, IL-6, as well as many chemokines, suggesting IL-17 develops the arthritis in cooperation with IL-6. Moreover, the expressions of IL-17-mediated NF-kB targets in the presence of an IL-6 signal are synergistically enhanced, particularly in type I collagen + cells such as fibroblasts and endothelial cells [Figures 1 and 2]. We named this IL-6-dependent NF-kB feedback loop the "inflammation amplifier." We defined the inflammation amplifier as the simultaneous stimulation effects of NF-kB and STAT3, two transcriptional factors in non-immune cells. This simultaneous stimulation induces the hyper-expression of various chemokines and IL-6, which are critical for the development of inflammation. Finally, results from disease model experiments showed that IL-6 acts as a fuel of the amplifier and the ef-

From the Laboratory of Developmental Immunology, JST-CREST, Graduate School of Frontier Biosciences, Graduate School of Medicine, and WPI Immunology Frontier Research Center, Osaka University, Osaka, Japan

Received: Mar. 13, 2013; Accepted: May 21, 2013

Correspondence to: Dr. Masaaki Murakami, Laboratory of Developmental Immunology, JST-CREST, Graduate School of Frontier Biosciences, Graduate School of Medicine, and WPI Immunology Frontier Research Center, Osaka University, Osaka, Japan. 2-2, Yamada-oka, Suita, Osaka, Japan 565-0871. Tel: 81-6-6879-3881; Fax: 81-6-6879-3889; E-mail: murakami@ molonc.med.osaka-u.ac.jp

DOI: $10.4103 / 2319-4170.113187$ 
fector molecules of the amplifier are various chemokines. Thus, we concluded that the inflammation amplifier is an inflammation inducer that compromises local homeostasis via an accumulation of various immune cells [Figure 2]. Indeed, we demonstrated that the inflammation amplifier is important for the pathogenesis of autoimmune diseases including arthritis in the F759 model, the multiple sclerosis model experimental autoimmune encephalomyelitis (EAE), and a chronic graft rejection model. ${ }^{[3-7]}$

\section{A gateway of immune cells in the CNS}

We have utilized an adoptive transfer method to induce EAE in which activated CD4+ T cells including Th17 cells are obtained from autoantigen-immunized mice. The autoreactive Th17 cells were enriched using an in vitro stimulation, isolated and infused into naïve recipient mice in which the CNS quiescence was maintained. In this adoptive transfer model, symptoms of a CNS-mediated disease were found from around 5 days after the intravenous pathogenic $\mathrm{T}$ cell transfer. This observation is difficult to explain when considering the blood-brain barrier (BBB), which strictly limits the inflow of substances like proteins and cells from the bloodstream into the CNS, thereby maintaining a homeostatic environment for surrounding neurons and glia cells. The BBB is formed and maintained by endothelial cells and corresponding tight junctions, which arise from claudins and occludins in collaboration with pericytes, macrophages, and astrocytes. ${ }^{[8,9]}$ We prepared whole-mount sections of adult mice using a macrotome and examined the accumulation of transferred cells throughout the body to identify where pathogenic Th17 cells breach the BBB. To our surprise, we found autoreactive Th17 cells preferentially accumulated in the fifth lumbar (L5) cord rather than the brain or other

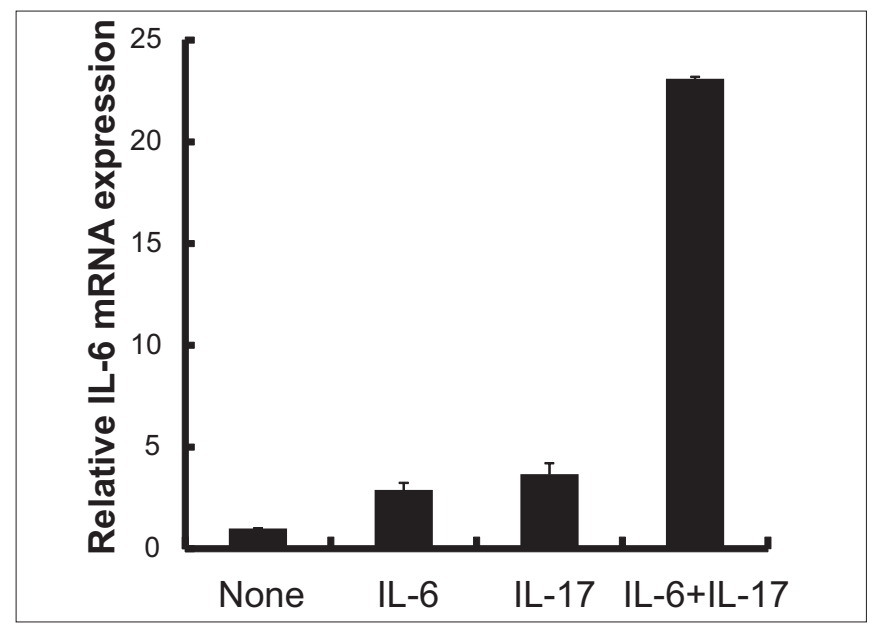

Figure 1: Synergistic effects of IL-17 and IL-6 costimulation on IL-6 mRNA expression. An endothelial cell line was stimulated with IL-17 and/or IL-6 for $3 \mathrm{~h}$, and IL-6 mRNA expression was determined by quantitative polymerase chain reaction. levels of the spinal cords at the earliest phase of EAE. ${ }^{[6]}$ This finding fits well with a typical clinical EAE sign in which the tail and lower body are first affected. We also found, using a supersensitive MRI, blood vessel tracks in L5 are altered due to the formation of edema in the L5 cord (manuscript in preparation). These results are in sharp contrast with those of Sallus et al., who reported that the choroid plexus, a specialized epithelial structure in the brain, expresses CCL20 constitutively to attract CCR6+ pathogenic Th17 cells. ${ }^{[10]}$ In this study, however, the CNS disease was induced by means of complete Freund's adjuvant (CFA), which is widely used for activating the immune system, particularly innate immunity, in animals. CFA is known as an inducer of inflammatory pain or fever, thus potentially affecting the pathophysiological status of the brain and the spinal cord. Moreover, they also used pertussis toxin, which may affect the endothelial cell activation and route of migration. On the other hand, we utilized the adoptive transfer model, which used neither CFA nor pertussis toxin, to induce EAE. Here, Th17 cells obtained from myelin oligodendrocyte glycoprotein (MOG)-immunized mice were infused into naïve recipients so that quiescence of the CNS is better preserved. We, therefore, concluded that the discrepancy between the results might be because of the induction method. Another factor may be the time course for the analyses, as we observed pathogenic $\mathrm{T}$ cell accumulation in brains including the choroid plexus at later times.

Further experiments revealed that the accumulation of pathogenic Th17 cells is restricted around the dorsal vessel of L5. Indeed, CCL20 mRNA levels were highest in the L5 dorsal vessel compared with vessels in other lumbar cords. Interestingly, even in naïve animals (those that did not receive the pathogenic T cell transfer), CCL20 as well

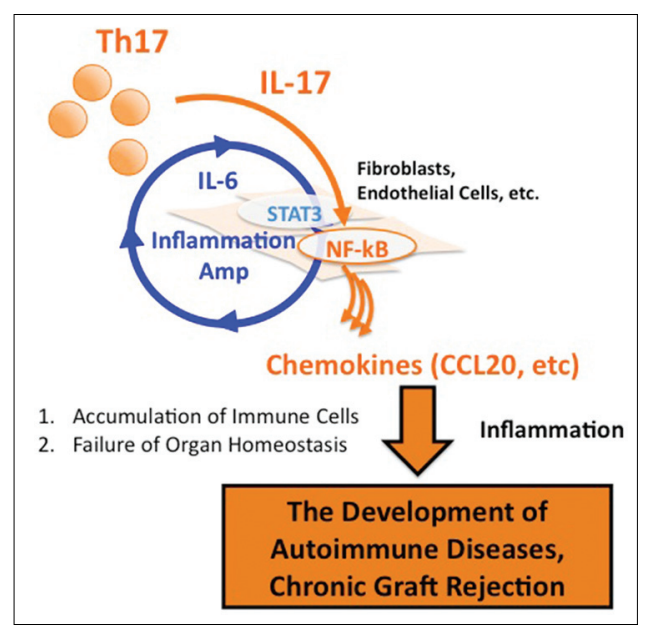

Figure 2: The inflammation amplifier contributes to many diseases and disorders. NF-kB stimulators such as IL-17 trigger the inflammation amplifier, a positive feedback loop for NF-kB targets including IL-6 and chemokines, through the IL-6-STAT3 pathway in non-immune cells. 
as many other chemokines were highly expressed in the L5 dorsal vessel. Moreover, using NF-kB reporter mice, NF-kB activity was found to be higher at L5 vessels than at vessels of L1 or the cervical cord. The elevated CCL20 level at L5 vessels was less in mutant mice that had defective inflammation amplifiers, such as IL-6-deficient mice. It is known that a small number of immune cells are present in the CNS even under normal conditions, suggesting that there exists a gateway for immune cells to enter this prohibited area. In this respect, it is tempting to speculate that a threshold activation of the inflammation amplifier at L5 dorsal vessels even at steady state creates this gate, although direct evidence for linking the inflammation amplifier and immune homeostasis in the CNS in the steady state is still lacking.

\section{The gateway theory}

We described earlier how an accumulation of pathogenic $\mathrm{T}$ cells is present at the dorsal vessels of L5 in the preclinical phase of EAE. Yet, why the accumulation happens at these vessels in unclear. One clue has come from studies that examined the gravitational effects on soleus muscles.

The dorsal root ganglia (DRG) of sensory neurons from soleus muscles are located beside L5. ${ }^{[11]}$ We, therefore, hypothesized that frequent stimulation of the soleus muscles by gravity will induce activation of the inflammation amplifier in L5 dorsal vessels via these sensory nerves. We employed the mouse tail-suspension model, a ground-based experimental model developed at the National Aeronautics and Space Administration, which orients the mouse such that the forelimbs touch the ground and the hind limbs are released from the gravity stimuli. The result was that pathogenic Th17 cells no longer accumulated at the L5 dorsal side; instead, they were seen at the cervical cords, indicating a new gateway was created via the strong gravity stimuli to the forelimbs. Consistent with this observation, the tail suspension significantly suppressed CCL20 expression in L5 dorsal blood vessels and decreased the expression of the neural activation marker, c-Fos, in L5 DRG. In addition, when soleus muscles were stimulated by electric pulses in tail-suspended mice, c-Fos expression as well as CCL20 expression and Th17 accumulation were all restored at the L5 cord. These results clearly demonstrate that regional neural activation via an anti-gravity response by the soleus muscles plays a role in activating the inflammation amplifier in L5 dorsal blood vessels, causing excess expression of chemokines and the recruitment of immune cells including pathogenic Th17 cells.

We next considered how afferent sensory activation induced by the soleus muscles alters the status of dorsal blood vessels at the L5 cord. Although a precise neural network has not been clarified, we showed the involvement of sympathetic nerves. We first measured blood flow speed in several vessels including those of L5. Indeed, blood flow speed at L5 dorsal vessels was slower when mice were tail-suspended, while electronic stimulations in the soleus muscles increased the speed. These results strongly suggest a contribution of automatic nerves including sympathetic ones. On the other hand, blood flow speeds in the other vessels like femoral vessels, brain surface vessels, and the portal vein were not affected by tail suspension. Moreover, activation of the sympathetic ganglion beside the L5 cord, but not the L1 cord, was activated. Furthermore, treatment with a norepinephrine receptor antagonist, atenolol, significantly suppressed CCL20 mRNA expression, NF-kB activation, and Th17 accumulation at the L5 vessels, and abrogated EAE development. Consistent with these in vivo results, norepinephrine stimulates the inflammation amplifier in endothelial cells based on IL-6 or CCL20 expressions in vitro [Figure 3] (data not shown). Thus, neural stimulation of the soleus muscles by gravity causes sympathetic nerve activation to produce norepinephrine, which creates a gateway into the CNS at L 5 dorsal vessels by promoting the excess activation of the inflammation amplifier [Figure 4]. Autoreactive pathogenic Th17 cells exploit this gateway to infiltrate the CNS and induce local inflammation by producing cytokines, which further enhances the inflammation amplifier activation in the parenchyma of the spinal cord.

Further, electronically stimulating thigh muscles including the quadriceps, which are regulated by sensory neurons from L3 DRG, increased the expression of the inflammation amplifier target chemokine, CCL20, in L3 dorsal vessels in mice. In a similar fashion, CCL20 chemokine levels in the fifth cervical to fifth thoracic dorsal vessels were upregulated by stimulations of the upper arm muscles including the epitrochlearis/triceps brachii, which are controlled by sensory

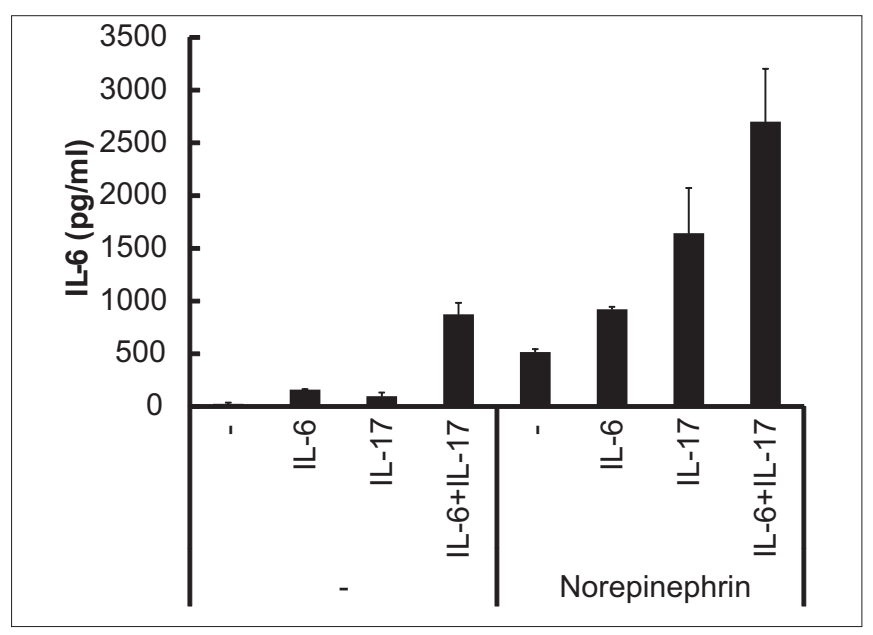

Figure 3: Synergistic effects of norepinephrine with IL-6 and/or IL-17 on the inflammation amplifier activation. An endothelial cell line was stimulated with IL-17, IL-6, and norepinephrine for $24 \mathrm{~h}$. The resulting IL-6 in culture supernatant was measured by enzyme-linked immunosorbent assay (ELISA) assay. A synergistic effect was found not only with IL-17 and IL-6 costimulation but also with norepinephrine and IL-17 and/or IL-6 costimulation. 


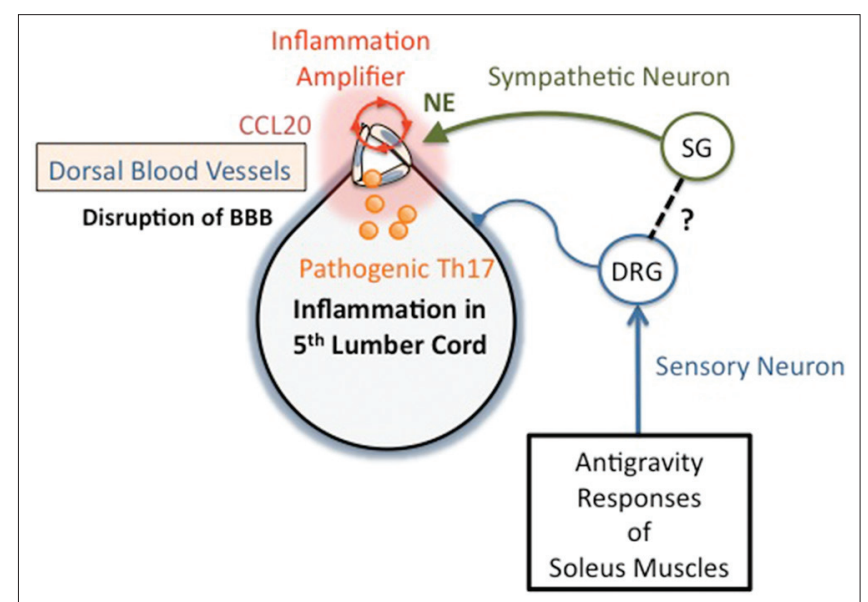

Figure 4: Regional neural activation regulates a gateway for immune cells. Antigravity responses by soleus muscles activate the inflammation amplifier at L5 dorsal blood vessels via sympathetic nerve activation. Released norepinephrine (NE) by a sympathetic nerve stimulates the inflammation amplifier at the dorsal vessels in endothelial cells to produce CCL20, a chemoattractant for pathogenic Th17 cells. The dorsal root ganglion (DRG) and sympathetic ganglion (SG) are located near the fifth lumbar cord.

neurons located at the corresponding areas [Figure 5]. ${ }^{[11]}$ Based on these results, we propose "the gateway theory," which describes how blood vessels act as a gateway that opens and closes based on regional neural stimulations to direct immune cell infiltration into the CNS. Consistent with this notion, we found that the expression of all observed chemokines increased in L5 vessels. These phenomena may suggest the importance of the L5 gateway for the accumulation of various immune cells in the CNS including not only pathogenic T cells but also some virus-specific ones. Investigations on whether the gate theory can be generally applied to various tissues other than the CNS are currently ongoing. Manipulation of the gateway should have several clinical benefits. For example, closing a specific gate could dampen autoimmune inflammation in the corresponding organ without any systemic immune suppression, while opening it in tumors may enhance cancer immunotherapy. The precise molecular mechanisms for gating are needed, however, before practical application of the gateway theory can begin.

\section{Conclusions}

In this review, we have attempted to summarize recent understandings of the inflammation amplifier, an NF-kB feedback loop, which depends on the simultaneous activation of NF-kB and STAT3, and its regulation of immunological homeostasis in vivo including the CNS [Figure 2]. Recent evidences have suggested that the inflammation amplifier is a key mechanism in the development of autoimmune diseases in the CNS by creating a gateway for immune cells including pathogenic $\mathrm{T}$ cells to bypass the BBB [Figure 4].

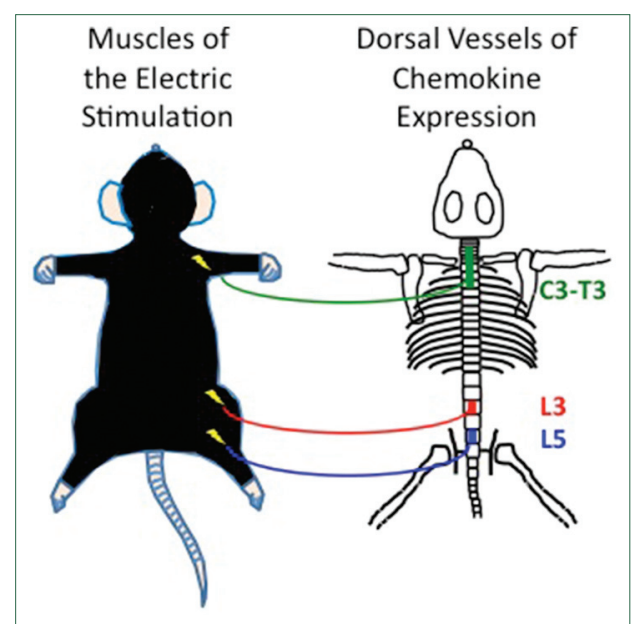

Figure 5: The gateway theory. Electric pulses to the upper arm, thigh, and soleus muscles induce activation of the inflammation amplifier (chemokine upregulation). This activation opens a gate in the dorsal vessels of the fifth cervical to fifth thoracic (C5-T5), third lumbar (L3), and fifth lumbar (L5) cords, indicating it as a possible target for the manipulation of immune cell migration in vivo.

We further introduce the gateway theory using our findings of neuro-immune interactions. This theory attempts to explain how immune cells at specific vessels in the spinal cords are regulated by regional neural activations and the inflammation amplifier. We believe that the gateway theory has great promise for therapeutic interventions of inflammatory diseases including autoimmune diseases, metabolic syndromes, neurodegenerative diseases, and other inflammatory diseases such as allergy and atopic dermatitis.

\section{REFERENCES}

1. Bettelli E, Oukka M, Kuchroo VK. T (H)-17 cells in the circle of immunity and autoimmunity. Nat Immunol 2007;8:345-50.

2. Nakae S, Nambu A, Sudo K, Iwakura Y. Suppression of immune induction of collagen-induced arthritis in IL-17-deficient mice. J Immunol 2003;171:6173-7.

3. Ogura H, Murakami M, Okuyama Y, Tsuruoka M, Kitabayashi C, Kanamoto $\mathrm{M}$, et al. Interleukin-17 promotes autoimmunity by triggering a positive-feedback loop via interleukin-6 induction. Immunity 2008;29:628-36.

4. Langrish CL, Chen Y, Bluemenschein WM, Mattson J, Basham B, Sedgwick JD, et al. IL-23 drives a pathogenic T cell population that induces autoimmune inflammation. J Exp Med 2005;201:233-40.

5. Murakami M, Okuyama Y, Ogura H, Asano S, Arima Y, Tsuruoka M, et al. Local microbleeding facilitates IL-6- and IL-17-dependent arthritis in the absence of tissue antigen recognition by activated T cells. J Exp Med 2011;208:103-14.

6. Arima Y, Harada M, Kamimura D, Park JH, Kawano F, Yull FE, et al. Regional neural activation defines a gateway for autoreactive $\mathrm{T}$ cells to cross the blood-brain barrier. Cell 2012;148:447-57.

7. Lee J, Nakagiri T, Oto T, Harada M, Morii E, Shintani Y, et al. IL-6 amplifier, NF-kB-triggered positive feedback for IL-6 signaling, 
in grafts is involved in allogenic rejection responses. J Immunol 2012;189:1928-36.

8. Abbott NJ, Ronnback L, Hansson E. Astrocyte-endothelial interactions at the blood-brain barrier. Nat Rev Neurosci 2006;7:41-53.

9. Steed E, Balda MS, Matter K. Dynamics and functions of tight junctions. Trends Cell Biol 2010;20:142-9.
10. Reboldi A, Coisne C, Baumjohann D, Benvenuto F, Bottinelli D, Lira S, et al. C-C chemokine receptor 6-regulated entry of TH-17 cells into the CNS through the choroid plexus is required for the initiation of EAE. Nat Immunol 2009;10:514-23.

11. Ohira Y, Kawano F, Stevens JL, Wang XD, Ishihara A. Load-dependent regulation of neuromuscular system. J Gravit Physiol 2004;11:127-8. 